\title{
FINITE GROUPS WITH SOME WEAKLY S-SUPPLEMENTED SUBGROUPS
}

\author{
WENBIN GUO \\ Department of Mathematics, University of Science and Technology of China, \\ Hefei 230026, China \\ e-mail:wbguo@ustc.edu.cn \\ K. P. SHUM \\ Department of Mathematics, The University of Hong Kong, \\ Pokfulam Road, Hong Kong, China \\ e-mail:kpshum@maths.hku.hk \\ and FENGYAN XIE \\ Humanistic Management College, Anyang Normal University, Anyang 455000, China \\ e-mail:kfxiefengyan@163.com
}

(Received 20 September 2008; accepted 1 July 2010; first published online 8 December 2010)

\begin{abstract}
Let $H$ be a subgroup of a group $G$. Then, we call $H$ weakly s-supplemented in $G$ if $G$ has a subgroup $T$ such that $H T=G$ and $H \cap T \leq H_{S G}$, where $H_{s G}$ is the largest s-permutable subgroup of $G$ contained in $H$. In this paper, we use the weakly s-supplemented subgroups to characterize the structure of groups. A series of known results in the literature are unified and generalized.
\end{abstract}

2010 Mathematics Subject Classification. 20D10, 20 D25.

1. Introduction. All groups $G$ considered in this paper are finite groups.

The structure of a group $G$ under the assumption that some primary subgroups of $G$ are well situated in $G$ has been investigated by many authors in the literature. For instance, Ito has proved that a group $G$ of odd order is nilpotent provided that all the minimal subgroups of $G$ lie in the centre of $G$ (see [6, III, Theorem 5.3]). An extension of Ito's results is the following statement: (1) For an odd prime $p$, if every subgroup of order $p$ lies in the centre of $G$, then $G$ is $p$-nilpotent; (2) if all elements of $G$ of order 2 or of order 4 lie in the centre of $G$, then $G$ is 2-nilpotent (see [6, IV, Theorem 5.5]). Along this direction, Buckley [2] proved that a group $G$ of odd order is supersolvable if every minimal subgroup of $G$ is normal in $G$. Some other generalizations have also been obtained by using the theory of formation and some generalized normal subgroups (see, for example, $[\mathbf{1}, \mathbf{4}, \mathbf{1 0}, \mathbf{1 7}, \mathbf{1 9}, \mathbf{2 2}]$ ).

Recall that a subgroup $H$ of a group $G$ is said to be permutable (or quasinormal) in $G$ if $H T=T H$ for any subgroup $T$ of $G$. A subgroup $H$ of a group $G$ is said to be s-permutable (or $\pi$-quasi-normal) in $G$ if $H P=P H$ for any Sylow subgroup $P$ of $G$. A subgroup $H$ of a group $G$ is said to be c-normal [16] (c-supplemented [18]) in $G$ if there exists a normal subgroup (a subgroup) $T$ of $G$ such that $H T=G$ and $H \cap T \leq H_{G}$, where $H_{G}$ is the normal core of $G$. On the other hand, Skiba [15] called a subgroup $H$ of a group $G$ weakly s-permutable in $G$ if $G$ has a subnormal subgroup $T$ such 
that $H \cap T \leq H_{s G}$, where $H_{s G}$ is the largest s-permutable subgroup of $G$ contained in $H$.

The following is the definition of a weakly s-supplemented subgroup introduced by Skiba in [15].

Definition 1.1 ([15, Definition 2.9]). Let $H$ be a subgroup of a group $G$. $H$ is said to be weakly s-supplemented in $G$ if $G$ has a subgroup $T$ such that $H T=G$ and $H \cap T \leq H_{S G}$, where $H_{S G}$ is the largest s-permutable subgroup of $G$ contained in $H$. In this case, $T$ is said to be a weakly s-supplement of $H$ in $G$.

It is easy to see that all normal subgroups, c-normal subgroups, c-supplemented subgroups, permutable subgroups, s-permutable subgroups and weakly s-permutable subgroup of $G$ are all weakly s-supplemented. The following examples show that the converse is not true.

EXAMPLE 1.2. Let $G=A_{5}=A_{4} C_{5}$. Then $C_{5}$ is weakly s-supplemented in $G$ since $C_{5} A_{4}=G$ and $C_{5} \cap A_{4}=1$. Obviously, $C_{5}$ is not normal, c-normal, permutable, s-permutable and also not weakly s-permutable in $G$.

ExAmple 1.3. Let $G=\langle a, b| a^{4}=1, a^{2}=b^{2}$ and $\left.b^{-1} a b=a^{-3}\right\rangle$. Then $\Phi(G)=$ $\left\langle a^{2}, b^{2}\right\rangle=\left\langle a^{2}\right\rangle \times\left\langle b^{2}\right\rangle$. Since $G$ is a 2 -group, $\left\langle b^{2}\right\rangle$ is s-permutable in $G$. In particular, $\left\langle b^{2}\right\rangle$ is weakly s-supplemented in $G$. However, $\left\langle b^{2}\right\rangle$ is not c-supplemented in $G$. In fact, $\left\langle b^{2}\right\rangle$ has only a supplemented subgroup $G$ in $G$, but $\left\langle b^{2}\right\rangle \cap G=\left\langle b^{2}\right\rangle$ is not normal in $G$.

In this paper, we shall use the weakly s-supplemented subgroups to describe the structures of some finite groups. A number of previously known results in the literatures are unified and generalized.

2. Preliminaries. Recall that a class of groups $\mathfrak{F}$ is a formation if $\mathfrak{F}$ is closed under homomorphic images and every group $G$ has a smallest normal subgroup (which is called the $\mathfrak{F}$-residual of $G$ and is denoted by $G^{\mathfrak{F}}$ ) whose quotient is in $\mathfrak{F}$. A formation $\mathfrak{F}$ is said to be s-closed if every subgroup of $G$ is in $\mathfrak{F}$ whenever $G \in \mathfrak{F}$. A formation $\mathfrak{F}$ is said to be saturated if it contains every group $G$ with $G / \Phi(G) \in \mathfrak{F}$. A map $f$ from the set of all primes to the set of all formations is said a formation function. A formation $\mathfrak{F}$ is said to be local if there exists a formation function $f$ such that $\mathfrak{F}=L F(f)$, where $L F(f)=\left\{G \mid G / C_{G}(H / K) \in f(p)\right.$ for all chief factors $H / K$ of $G$ and every $\left.p \in \pi(H / K)\right\}$. It is well known that a formation $\mathfrak{F}$ is saturated if and only if $\mathfrak{F}$ is local.

In this paper, we denote by $\mathfrak{N}$ the class of the nilpotent groups, and by $\mathfrak{H}_{p}$ the class of the $p$-nilpotent groups. It is well known that both $\mathfrak{N}$ and $\mathfrak{H}_{p}$ are s-closed saturated formations.

Let $\mathfrak{F}=L F(f)$ is a saturated formation. A chief factor $H / K$ of a group $G$ is said to be $f$-central in $G$ (see [3] or [5, definition 2.4.3]) if $G / C_{G}(H / K) \in f(p)$. The symbol $Z_{\infty}^{\mathfrak{F}}(G)$ denotes the $\mathfrak{F}$-hypercentre of a group $G$, that is, it is the product of all normal subgroups of $G$ whose $G$-chief factors are $f$-central. A subgroup $H$ is said to be $\mathfrak{F}$-hypercentral in $G$ if $H \leq Z_{\infty}^{\mathfrak{F}}(G)$. If $\mathfrak{F}=\mathfrak{N}$, then $Z_{\infty}^{\mathfrak{N}}(G)$ is precisely the hypercentre $Z_{\infty}(G)$ of $G$.

Let $p$ be a prime and $G$ a group. Then we write $\mathcal{P}_{p}(G)=\{x \in G|| x \mid=p\} ; \mathcal{P}_{4}(G)=$ $\{x \in G|| x \mid=4\} ; \mathcal{P}_{p}^{*}(G)=\{x \in G|| x \mid=p$ or $|x|=4\} ; \mathcal{P}(G)=\cup_{p \in \pi(G)} \mathcal{P}_{p}(G)$.

For notations and terminologies not mentioned in this paper, the reader is referred to $[3,5,14]$. 
For the sake of convenience, we first cite some known results in the literature which will be useful in the following.

LeMma 2.1 [20, Theorem I.6.1]. Let $G$ be a group, $H \leq G$ and $H$ s-permutable in G. Then

(1) if $H \leq K \leq G$, then $H$ is s-permutable in $K$.

(2) if $\theta$ is a homomorphism of $G$, then $H^{\theta}$ is s-permutable in $G^{\theta}$.

LEMMA 2.2 [21, Lemma 2.2]. Let $G$ be a group. If $H$ is a p-subgroup of $G$ for some prime $p$ and $H$ is s-permutable in $G$, then the following properties hold:

(1) $H \leq O_{p}(G)$.

(2) $O^{p}(G) \leq N_{G}(H)$.

LEMMA 2.3 [4, Lemma 5]. Let $\mathfrak{F}$ be an s-closed saturated formation and $H$ a subgroup of a group $G$. Then $H \cap Z_{\infty}^{\mathfrak{F}}(G) \subseteq Z_{\infty}^{\mathfrak{F}}(H)$.

Lemma 2.4. Let $G$ be a group, $H \leq G$ and $H$ be weakly s-supplemented in $G$. Then

(1) if $H \leq K \leq G$, then $H$ is weakly s-supplemented in $K$.

(2) if $N \unlhd G$ and $N \leq H$, then $H / N$ is weakly s-supplemented in $G / N$.

(3) if $N \unlhd G$ and $(|N|,|H|)=1$, then $H N / N$ is weakly s-supplemented in $G / N$.

(4) if $N / \Phi(N)$ is a soluble chief factor of $G$ and $H \leq N$, then $H$ is s-permutable in $G$.

Proof. For the proofs of statements (1)-(3), the reader can be referred to [15, Theorem 2.10]. We now prove statement (4). Since $H$ is weakly s-supplemented in $G$, there exists a subgroup $T$ of $G$ such that $H T=G$ and $H \cap T \leq H_{S G}$. Let $N_{1}=N \cap T$. Then $N_{1}$ is normal in $T$ and so $N_{1} \Phi(N) / \Phi(N)$ is normal in $T \Phi(N) / \Phi(N)$. Since $N / \Phi(N)$ is a soluble chief factor of $G, N / \Phi(N)$ is an elementary abelian group and consequently, $N_{1} \Phi(N) / \Phi(N)$ is normal in $N / \Phi(N)$. This shows that $N_{1} \Phi(N) / \Phi(N)$ is a normal subgroup of $G / \Phi(N)$. Hence, $N_{1} \Phi(N) / \Phi(N)=1$ or $N_{1} \Phi(N) / Z \Phi(N)=$ $N / \Phi(N)$. If $N_{1} \Phi(N) / \Phi(N)=1$, then $N=N \cap H T=H(N \cap T)=H$ since $H \leq N$. This means that $H$ is normal in $G$ and thereby $H$ is s-permutable in $G$. If $N_{1} \Phi(N) / \Phi(N)=N / \Phi(N)$, then $T=G$ and so $H=H \cap T \subseteq H_{S G}$. This implies that $H=H_{s G}$ is s-permutable.

LEMMA 2.5. Let $G$ be a group and $p$ a prime such that $p^{n+1} \nmid|G|$ for some integer $n \geq 1$. If $\left(|G|,(p-1)\left(p^{2}-1\right) \ldots\left(p^{n}-1\right)\right)=1$, then $G$ is p-nilpotent.

Proof. Suppose that the assertion is false and let $G$ be a counterexample of minimal order. It is obvious that every subgroup of $G$ satisfies the hypothesis of the lemma. The minimal choice of $G$ implies that $G$ is a minimal non- $p$-nilpotent group. By [13, Theorem 10.3.3] and [5, Theorem 3.4.11], $G=[P] Q$ is a subdirect product of a Sylow $p$-subgroup $P$ of $G$ and a Sylow $q$-subgroup $Q$ of $G$ for some primes $p, q \in \pi(G)$. It is easy to see that every proper quotient group of $G$ satisfies the hypothesis. Thus, $\Phi(P)=\Phi(G)=1$ and so $P$ is an elementary abelian $p$-group. Since $N_{G}(P) / C_{G}(P)$ is isomorphic to a subgroup of $\operatorname{Aut}(P)$ and $|\operatorname{Aut}(P)|$ divides $p^{\frac{n(n-1)}{2}}(p-1)\left(p^{2}-1\right) \cdots\left(p^{n}-1\right)$ for $|P| \leqslant$ $p^{n}, N_{G}(P) / C_{G}(P)=1$. This result induces that $G$ is $p$-nilpotent by the well-known Burnside's theorem (see [13, Theorem 10.1.8]). This contradiction completes the proof. 
Recall that the generalized Fitting subgroup $F^{*}(G)$ of a group $G$ is the product of all normal quasi-nilpotent subgroups of $G$. We shall need the following well-known facts of the generalized Fitting subgroup (see [7, Chapter X]) to prove our new results.

LEMMA 2.6. Let $G$ be a group. Then the following statements hold:

(1) If $N$ is a normal subgroup of $G$, then $F^{*}(N) \leq F^{*}(G)$.

(2) $F(G) \leq F^{*}(G)=F^{*}\left(F^{*}(G)\right)$. If $F^{*}(G)$ is soluble, then $F^{*}(G)=F(G)$.

(3) If $N$ is a normal subgroup of $G$ and $N \leq F^{*}(G)$, then $F^{*}(G) / N \leq F^{*}(G / N)$.

(4) If $N$ is a normal subgroup of $G$ and $N \leq Z(G)$, then $F^{*}(G) / N=F^{*}(G / N)$.

\section{Main results.}

LEMMA 3.1. Let $p$ be a prime and $G$ a group with $\left(|G|,(p-1)\left(p^{2}-1\right) \cdots\left(p^{n}-\right.\right.$ $1))=1$, for some integer $n \geq 1$. Suppose that there exists a subgroup $D$ of $G$ with order $p^{n}$ such that all the subgroups $H$ of $G$ with $|H|=|D|$ or $|H|=2|D|$ (if the Sylow p-subgroup $P$ of $G$ is a non-abelian 2-group and $|P: D|>2$ ) not having a p-nilpotent supplement in $G$ are weakly s-supplemented in $G$, then $G$ is p-nilpotent.

Proof. Suppose that the statement is false and let $G$ be a counterexample of minimal order. Then $p^{n+1}|| G \mid$ by Lemma 2.5 . We proceed the proof by the following steps.

(1) Every proper subgroup of $G$ is p-nilpotent.

Let $L$ be a proper subgroup of $G$. Then $\left(|L|,(p-1)\left(p^{2}-1\right) \cdots\left(p^{n}-1\right)\right)=1$. If $p^{n+1} \nmid|L|$, then by Lemma $2.5, L$ is $p$-nilpotent. Now assume that $p^{n+1}|| L \mid$. Let $D_{1}$ be a subgroup of $L$ of order $p^{n}$ and $H$ a subgroup of $L$ with $|H|=\left|D_{1}\right|$ or $|H|=2\left|D_{1}\right|$. Then by the hypothesis, $H$ has a $p$-nilpotent supplement $T$ in $G$ or is weakly s-supplemented in $G$. In the former case, $L=L \cap H T=H(L \cap T)$ and $L \cap T$ is a $p$-nilpotent supplement of $H$ in $L$. In the latter case, by Lemma 2.4(1), $H$ is weakly s-supplemented in $L$. This shows that $L$ satisfies our hypothesis. The minimal choice of $G$ implies that $L$ is $p$-nilpotent.

(2) G has a normal Sylow p-subgroup P satisfying the following properties:

(i) $G=[P] Q$, where $Q$ is a Sylow q subgroup of $G$;

(ii) $P / \Phi(P)$ is a chief factor of $G$;

(iii) If $P$ is abelian, then $P$ is an elementary abelian group;

(vi) $\exp (P)=p$ or $\exp (P)=4$.

In fact, by(1), $G$ is a minimal non- $p$-nilpotent. Hence (2) holds by [13, (10.3.3)] and [5, Theorem 3.4.12].

(3) $P$ is not cyclic.

Suppose that $P$ is cyclic. If $\exp (P)=p$, then $|P|=p$ and so $|\operatorname{Aut}(P)|=p-1$. If $\exp (P)=4$, then $|P|=4$ and so $|\operatorname{Aut}(P)|=2$. It is well known that $N_{G}(P) / C_{G}(P)$ is isomorphic to some subgroup of $\operatorname{Aut}(P)$. Since $P \subseteq C_{G}(P)$ and $(|G|, p-1)=1$, $N_{G}(P) / C_{G}(P)=1$. Thus, by Burnside's theorem, $G$ is $p$-nilpotent. This contradiction shows that $P$ is not cyclic.

(4) Let $H$ be a subgroup of $P$ with $|H|=|D|$ or $|H|=2|D|$ (when $P$ is a non-abelian 2-group and $|P: D|>2)$; then $H$ is s-permutable in $G$.

Let $T$ be any supplement of $H$ in $G$. Then $H T=G$ and so $P=P \cap H T=H(P \cap$ $T)$. Since $P / \Phi(P)$ is the chief factor of $G, P / \Phi(P)$ is an elementary abelian $p$-group and hence $(P \cap T) \Phi(P) / \Phi(P)$ is normal in $P / \Phi(P)$. Now, since $(P \cap T) \Phi(P) / \Phi(P)$ is normal in $T \Phi(P) / \Phi(P),(P \cap T) \Phi(P) / \Phi(P)$ is normal in $G / \Phi(P)$. It follows that $P \cap T \subseteq \Phi(P)$ or $P \cap T=P$. If $P \cap T \subseteq \Phi(P)$, then $H=P$ is normal in $G$, which contradicts (2). If $P \cap T=P$, then $T=G$ is not $p$-nilpotent. Thus, by the hypothesis, 
$H$ is weakly s-supplemented in $G$. It follows from Lemma 2.4(4) that $H$ is s-permutable in $G$. Therefore, (4) holds.

(5) $|P: D|>p$.

Suppose that $|P: D|=p$. Then $|P|=p^{n+1}$ and so every maximal subgroup $H$ of $P$ is s-permutable in $G$ by the hypothesis and (4). This induces that $H Q$ is a proper subgroup of $G$ and hence $H Q$ is $p$-nilpotent. Thus, $Q$ is normal in $H Q$. It follows from (3) that $Q$ is normal in $G$, a contradiction. Therefore, $|P: D|>p$.

(6) Final contradiction.

By our hypothesis and (4), all subgroups $H$ of $P$ with $|H|=|D|$ or $|H|=2|D|$ (when $P$ is a non-abelian 2-group and $|P: D|>2$ ) are s-permutable $G$. Then by (5), the subgroup $H Q$ is a proper subgroup of $G$ for any such subgroup $H$. Hence $H Q$ is $p$-nilpotent. This implies that $Q$ is normal in $H Q$. It follows from (3) that $Q$ is normal in $G$. This final contradiction completes the proof.

COROLlARY 3.2. Let $p$ be a prime and $G$ a group with $\left(|G|,(p-1)\left(p^{2}-1\right) \cdots\left(p^{n}-\right.\right.$ $1))=1$, for some integer $n \geq 1$. Suppose that there exists a subgroup $D$ of $G$ such that $1<|D|<p^{n+1}$ and all subgroups $H$ of $G$ with $|H|=|D|$ or $|H|=2|D|$ (when the Sylow p-subgroup $P$ of $G$ is a non-abelian 2-group and $|P: D|>2$ ) not having a p-nilpotent supplement in $G$ are weakly s-supplemented in $G$. Then $G$ is p-nilpotent.

THEOREM 3.3. Let $p$ be a prime and $\mathfrak{F}$ a saturated formation containing $\mathfrak{H}_{\mathfrak{p}}$. Suppose that $G$ is a group with $\left(|G|,(p-1)\left(p^{2}-1\right) \cdots\left(p^{n}-1\right)\right)=1$, for some integer $n \geq 1$. Then $G \in \mathfrak{F}$ if and only if $G$ has a normal subgroup $E$ such that $G / E \in \mathfrak{F}$ and there exists a subgroup $D$ of $E$ such that $1<|D|<p^{n+1}$ and all subgroups $H$ of $E$ with $|H|=|D|$ or $|H|=2|D|$ (when the Sylow p-subgroup $P$ of $E$ is a non-abelian 2-group and $|P: D|>2$ ) not having a p-nilpotent supplement in $G$ are weakly s-supplemented in $G$.

Proof. The necessity part is obvious. We need only to prove the sufficiency part. Suppose that the statement is false and let $G$ be a counterexample of minimal order. Obviously, $\left(|E|,(p-1)\left(p^{2}-1\right) \cdots\left(p^{n}-1\right)\right)=1$ and either $H$ has a $p$-nilpotent supplement in $E$ or $H$ is weakly s-supplemented in $E$ by Lemma 2.4(1). Now, Corollary 3.2 implies that $E$ is $p$-nilpotent. Let $P$ be a Sylow $p$-subgroup of $E$ and $T$ a normal Hall $p^{\prime}$-subgroup of $E$. Then $T$ is normal in $G$. We now proceed to prove the theorem via the following steps.

(1) $T=1$.

If $T \neq 1$, then we first claim that $G / T$ (with respect to $E / T$ ) satisfies the hypothesis of the theorem. In fact, $(G / T) /(E / T) \simeq G / E \in \mathfrak{F}$. Let $N / T$ be an arbitrary subgroup of $E / T$ with $|N / T|=|D T / T|$ or $|N / T|=2|D T / T|$. Then $N=[T] L$, where $L$ is a Sylow $p$-subgroup of $N$. Thus, $|L|=|D|$ or $|L|=2|D|$. By the hypothesis, either $L$ has a $p$-nilpotent supplement $M$ in $G$ or $L$ is weakly s-supplemented in $G$. This means that either $N / T=T L / T$ has a $p$-nilpotent supplement $M T / T \simeq M / T \cap M$ in $G / T$ or $N / T$ is weakly s-supplemented in $G / T$ by Lemma 2.4(3). Hence, $G / T$ satisfies the hypothesis. The minimal choice of $G$ implies that $G / T \in \mathfrak{F}$. Let $f_{i}(\mathrm{i}=1,2)$ be a full and integrated formation functions such that $\mathfrak{H}_{\mathfrak{p}}=L F\left(f_{1}\right)$ and $\mathfrak{F}=L F\left(f_{2}\right)$, respectively. Since $T$ is a normal $p^{\prime}$-subgroup of $G, G / C_{G}\left(T_{i+1} / T_{i}\right) \in f_{1}(q)$ for every chief factor $T_{i+1} / T_{i}$ of $G$ with $T_{i+1} \leq T$ and every prime $q$ dividing $\left|T_{i+1} / T_{i}\right|$ (see, [5, p 98, Example 2]). Since $\mathfrak{H}_{\mathfrak{p}} \subseteq \mathfrak{F}, f_{1}(q) \subseteq f_{2}(q)$ by [5, Corollary 3.1.16]. It follows that $G / C_{G}\left(T_{i+1} / T_{i}\right) \in f_{2}(q)$. Therefore, $G \in \mathfrak{F}$ by $G / T \in \mathfrak{F}$. This contradiction shows that $T=1$. 
(2) Suppose that $Q$ is a Sylow $q$-subgroup of $G$, where $q \neq p$ is a prime divisor of $|G|$. Then $P Q=P \times Q$.

By (1), $P=E \unlhd G$. Hence, $P Q$ is a subgroup of $G$. Obviously, $D$ is a subgroup of $P Q$ and all subgroups $H$ of $P Q$ with $|H|=|D|$ or $|H|=2|D|$ (when $P$ is a non-abelian 2-group and $|P: D|>2$ ) not having a $p$-nilpotent supplement in $P Q$ are weakly ssupplemented in $P Q$ by Lemma 2.4(1). Hence by Corollary 3.2, $P Q$ is $p$-nilpotent. It follows that $Q \unlhd P Q$ and so $P Q=P \times Q$.

(3) Final contradiction.

Let $M$ be an arbitrary non-identity normal subgroup of $G$ contained in $P$ and $G_{p}$ a Sylow $p$-subgroup of $G$. By (2), we have $M Q=M \times Q$ for any Sylow $q$-subgroup of $G$. This induces that $O^{p}(G) \leq C_{G}(M)$ and $[M, G]=\left[M, G_{p} O^{p}(G)\right]=\left[M, G_{p}\right] \unlhd G$. Since $\left[M, G_{p}\right]<M$, there exists a normal subgroup $N$ of $G$ such that $M / N$ is a chief factor of $G$ and $[M, G] \leq N$. This implies that $M / N \leq Z(G / N)$. Let $f$ be the full and integrated formation function such that $\mathfrak{F}=L F(f)$. Then $G / C_{G}(M / N)=1 \in f(p)$. The arbitrary choice of $M$ implies that there exists a normal chain of $G$ contained in $P$ such that every chief factor $M / N$ is $f$-central. It follows that $G \in \mathfrak{F}$. The final contradiction completes the proof.

REMARK 3.4: The sufficiency of Theorem 3.3 would be false in general if the condition " $|H|=2|D|$ " is removed. For example, if we let $H=\langle a, b| a^{4}=1, a^{2}=$ $b^{2}$ and $\left.b^{-1} a b=a^{-1}\right\rangle$ to be a quaternion group with $G=[H]\langle\alpha\rangle$, where $\alpha$ is an automorphism of $H$ of order 3. Let $p=2$ and $n=1$. Then it is not difficult to show that $\left\langle a^{2}\right\rangle$ is a unique subgroup of $G$ with order 2 and $\left\langle a^{2}\right\rangle$ is normal in $G$. Hence, $\left\langle a^{2}\right\rangle$ is weakly s-supplemented in $G$. But it is obvious that $G$ is not a 2-nilpotent group.

LEMMA 3.5. Let $G$ be a group and $p$ a prime factor of $|G|$. Suppose that every element of $\mathcal{P}_{p}(G)$ is contained in $Z_{\infty}(G)$ and $\langle x\rangle$ is weakly s-supplemented in $G$ for every $x \in \mathcal{P}_{4}(G)$. Then $G$ is p-nilpotent.

Proof. Suppose that the statement is false and let $G$ be a counterexample of minimal order. Then we can prove the following facts:

(1) Every proper subgroup of $G$ is p-nilpotent.

Suppose that $H$ is a proper subgroup of $G$. Let $x \in \mathcal{P}_{p}(H)$. Then by the hypothesis, $x \in Z_{\infty}(G)$. By Lemma 2.3, $x \in Z_{\infty}(G) \cap H \subseteq Z_{\infty}(H)$. Let $x \in \mathcal{P}_{4}(H)$. By the hypothesis, $\langle x\rangle$ is weakly s-supplemented in $G$. Then by Lemma 2.4(1), $\langle x\rangle$ is weakly s-supplemented in $H$. Thus, the hypothesis holds for $H$. The minimal choice of $G$ implies that $H$ is $p$-nilpotent.

(2) $G=[P] Q$ is a subdirect product of a Sylow p-subgroup $P$ of $G$ and a Sylow $q$-subgroup $Q$ of $G, P / \Phi(P)$ is a chief factor of $G$ and $\exp (P)=p$ or $\exp (P)=4$.

By (1), $G$ is a minimal non- $p$-nilpotent. Hence (2) holds by $[\mathbf{1 3},(10.3 .3)]$ and $[\mathbf{5}$, Theorem 3.4.11].

(3) $\exp (P)=4$.

Suppose that $\exp (P)=p$. Then by the hypothesis, $P \subseteq Z_{\infty}(G)$ and consequently $G / Z_{\infty}(G) \simeq(G / P) /\left(Z_{\infty}(G) / P\right)$ is $p$-nilpotent. It follows that $G$ is $p$-nilpotent, a contradiction.

(4) $|x|=4$, for every $x \in P \backslash \Phi(P)$.

Suppose that there exists an element $x \in P \backslash \Phi(P)$ of order 2. Let $T=\langle x\rangle^{G}$. Then $T \leq P$ and $T \Phi(P) / \Phi(P)$ is normal in $G / \Phi(P)$. Since $P / \Phi(P)$ is a chief factor of $G$, $P=T$ and so $\exp (P)=2$, which contradicts (3). 
(5) $P$ is not cyclic.

Suppose that $P$ is cyclic. Then by (3), we know $|P|=4$ and hence $|\operatorname{Aut}(P)|=2$. It is well known that $N_{G}(P) / C_{G}(P)$ is isomorphic to some subgroup of $\operatorname{Aut}(P)$. Since $P \subseteq$ $C_{G}(P), N_{G}(P) / C_{G}(P)=1$. By Burnside's theorem, $G$ is $p$-nilpotent, a contradiction. Therefore $P$ is not cyclic.

(6) Final contradiction.

By (4) and the hypothesis, $\langle x\rangle$ is weakly s-supplemented in $G$ for every $x \in P \backslash \Phi(P)$. Then by Lemma 2.4(4), $\langle x\rangle$ is s-permutable in $G$. Hence $\langle x\rangle Q$ is a proper subgroup of $G$ by (2) and (4). Therefore, $\langle x\rangle Q$ is $p$-nilpotent and so $Q$ is normal in $\langle x\rangle Q$, for every $x \in P \backslash \Phi(P)$. It follows that $Q$ is normal in $G$. The final contradiction completes the proof.

COROLlary 3.6. Let $G$ be a group. Suppose that every element of $\mathcal{P}(G)$ is contained in $Z_{\infty}(G)$ and $\langle x\rangle$ is weakly s-supplemented in $G$ for every $x \in \mathcal{P}_{4}(G)$. Then $G$ is nilpotent.

PROPOSITION 3.7. Let $\mathfrak{F}$ be a saturated formation containing $\mathfrak{N}$. Suppose that a group $G$ has a normal subgroup $E$ such that $G / E \in \mathfrak{F}$ and $\langle x\rangle$ is weakly s-supplemented in $G$ for every $x \in \mathcal{P}_{4}(E)$. Then $G \in \mathfrak{F}$ if and only if every element of $\mathcal{P}(E)$ is contained in $Z_{\infty}^{\mathfrak{F}}(G)$.

Proof. The necessity is obvious. We need only to prove the sufficiency. Suppose that the statement is false and let $G$ be a counterexample of minimal order. We now proceed to prove the theorem via the following steps.

(1) $G^{\mathfrak{F}}$ is a p-group for some prime $p$ and $G^{\mathfrak{F}}$ satisfies the following conditions: (i) $G^{\mathfrak{F}} / \Phi\left(G^{\mathfrak{F}}\right)$ is a chief factor of $G$. (ii) $\exp \left(G^{\mathfrak{F}}\right)=p$ or $\exp \left(G^{\mathfrak{F}}\right)=4$ (if $|p|=2$ and $G^{\mathfrak{F}}$ is non-abelian). (iii) If $G^{\mathfrak{F}}$ is abelian, then $G^{\mathfrak{F}}$ is an elementary abelian group.

Since $G / E \in \mathfrak{F}, G^{\mathfrak{F}} \subseteq E$. Let $x \in \mathcal{P}\left(G^{\mathfrak{F}}\right)$. Then by the hypothesis, $x \in Z_{\infty}^{\mathfrak{F}}(G)$. By [5, Corollary 3.2.9], $Z_{\infty}^{\mathfrak{F}}(G) \cap G^{\mathfrak{F}} \subseteq Z\left(G^{\mathfrak{F}}\right) \subseteq Z_{\infty}\left(G^{\mathfrak{F}}\right)$ and so $x \in Z_{\infty}\left(G^{\mathfrak{F}}\right)$. Let $x \in$ $\mathcal{P}_{4}\left(G^{\mathfrak{F}}\right)$. By the hypothesis, $\langle x\rangle$ is weakly s-supplemented in $G$. Then by Lemma 2.4(1), $\langle x\rangle$ is weakly s-supplemented in $G^{\mathfrak{F}}$. Corollary 3.6 implies that $G^{\mathfrak{F}}$ is nilpotent.

Since $\mathfrak{F}$ is a saturated formation, there exists a maximal subgroup $M$ of $G$ such that $M G^{\mathfrak{F}}=G$. Let $Z=Z_{\infty}^{\mathfrak{F}}(G) \cap M$. We claim that $Z \subseteq Z_{\infty}^{\mathfrak{F}}(M)$. In fact, since $\left[Z_{\infty}^{\mathfrak{F}}(G), G^{\mathfrak{F}}\right]=1$ (see [5, Corollary 3.2.9]), $Z \subseteq C_{G}\left(G^{\mathfrak{F}}\right)$. This induces that every $G$ chief factor $H / K$ contained in $Z$ is also an $M$-chief factor and $G^{\mathfrak{F}} \subseteq C_{G}(H / K)$. Hence $M / C_{M}(H / K) \simeq M C_{G}(H / K) / C_{G}(H / K) \simeq G / C_{G}(H / K)$. Consequently, $Z \subseteq$ $Z_{\infty}^{\mathfrak{F}}(M)$. Since $M / M \cap G^{\mathfrak{F}} \cong M G^{\mathfrak{F}} / G^{\mathfrak{F}}=G / G^{\mathfrak{F}} \in \mathfrak{F}, M^{\mathfrak{F}} \subseteq G^{\mathfrak{F}}$. This implies that every element of $\mathcal{P}\left(M^{\mathfrak{F}}\right)$ is contained in $Z_{\infty}^{\mathfrak{F}}(M)$. Hence, $M$ (with respect to $M^{\mathfrak{F}}$ ) satisfies the hypothesis. The minimal choice of $G$ implies that $M \in \mathfrak{F}$.

Now by using [5, Theorem 3.4.2], we see that (1) holds.

(2) $\exp \left(G^{\mathfrak{F}}\right)=4$ and $|x|=4$ for $x \in P \backslash \Phi(P)$.

This can be obtained by using the same similar argument in Lemma 3.5(2-3).

(3) $\left|G^{\mathfrak{F}} / \Phi\left(G^{\mathfrak{F}}\right)\right|=2$.

Suppose that any subgroup $T / \Phi\left(G^{\mathfrak{F}}\right)$ of $G^{\mathfrak{F}} / \Phi\left(G^{\mathfrak{F}}\right)$ with order 2 is not normal in $G / \Phi\left(G^{\mathfrak{F}}\right)$. Obviously, $T=\langle x\rangle \Phi\left(G^{\mathfrak{F}}\right)$ for some $x \in T \backslash \Phi\left(G^{\mathfrak{F}}\right)$. By (2) and our hypothesis, $\langle x\rangle$ is weakly s-supplemented in $G$. Using Lemma 2.4(4), $\langle x\rangle$ is s-permutable in $G$. It follows from Lemma 2.1 that $T / \Phi\left(G^{\mathfrak{F}}\right)$ is s-permutable in $G / \Phi\left(G^{\mathfrak{F}}\right)$. Hence by Lemma $2.2, O^{2}\left(G / \Phi\left(G^{\mathfrak{F}}\right)\right) \leq N_{G / \Phi\left(G^{\mathfrak{F}}\right)}\left(T / \Phi\left(G^{\mathfrak{F}}\right)\right)$ and so $\mid G / \Phi\left(G^{\mathfrak{F}}\right): N_{G / \Phi\left(G^{\mathfrak{F}}\right)}\left(T / \Phi\left(G^{\mathfrak{F}}\right) \mid=2^{\alpha}\right.$ for some positive integer $\alpha$. This shows that the number of all subgroups of $G^{\mathfrak{F}} / \Phi\left(G^{\mathfrak{F}}\right)$ with order 2 is even, which contradicts [6, Theorem III, 8.5]. The contradiction shows that there exists a subgroup $T / \Phi\left(G^{\mathfrak{F}}\right)$ of $G^{\mathfrak{F}} / \Phi\left(G^{\mathfrak{F}}\right)$ with order 2 which is normal in $G / \Phi\left(G^{\mathfrak{F}}\right)$. But since $G^{\mathfrak{F}} / \Phi\left(G^{\mathfrak{F}}\right)$ is a chief factor of $G, G^{\mathfrak{F}} / \Phi\left(G^{\mathfrak{F}}\right)=T / \Phi\left(G^{\mathfrak{F}}\right)$. Consequently,$\left|G^{\mathfrak{F}} / \Phi\left(G^{\mathfrak{F}}\right)\right|=2$. 
(4) Final contradiction.

By (3), $G^{\mathfrak{F}} / \Phi\left(G^{\mathfrak{F}}\right)=\langle x\rangle \Phi\left(G^{\mathfrak{F}}\right) / \Phi\left(G^{\mathfrak{F}}\right)$ for some $x \in G^{\mathfrak{F}}$. It follows that $G^{\mathfrak{F}}=\langle x\rangle$. Then by (1), $G^{\mathfrak{F}}$ is an elementary abelian group, which contradicts (2). This final contradiction completes the proof.

LEMMA 3.8. Let $G$ be a group. Suppose that every element of $\mathcal{P}\left(F^{*}(G)\right)$ is contained in $Z_{\infty}(G)$ and $\langle x\rangle$ is weakly s-supplemented in $G$ for every $x \in \mathcal{P}_{4}\left(F^{*}(G)\right)$. Then $G$ is nilpotent.

Proof. Suppose that the statement is false and let $G$ be a counterexample of minimal order. By Lemma 2.3, we know that $Z_{\infty}(G) \cap F^{*}(G) \subseteq Z_{\infty}\left(F^{*}(G)\right)$. Then by the hypothesis, every element of $\mathcal{P}\left(F^{*}(G)\right)$ is contained in $Z_{\infty}\left(F^{*}(G)\right)$. On the other hand, by Lemma 2.4(1), $\langle x\rangle$ is weakly s-supplemented in $F^{*}(G)$ for every $x \in$ $\mathcal{P}_{4}\left(F^{*}(G)\right)$. Corollary 3.6 implies that $F^{*}(G)$ is nilpotent. Consequently, $F^{*}(G)=F(G)$. Let $F^{*}(G)=F, p$ be the smallest prime dividing $|F|$ and $P$ a Sylow $p$-subgroup of $F$. We now proceed the proof by proving the following claims.

(1) $G^{\mathfrak{N}}=G$, that is, $G / N$ is not nilpotent for any proper normal subgroup $N$ of $G$.

By Lemma 2.6(1), $F^{*}\left(G^{\mathfrak{N}}\right) \leq F$. By Lemma 2.3, $Z_{\infty}(G) \cap G^{\mathfrak{N}} \leq Z_{\infty}\left(G^{\mathfrak{N}}\right)$. Hence, by the hypothesis and Lemma $2.4(1)$, we see that every element of $\mathcal{P}\left(F^{*}\left(G^{\mathfrak{N}}\right)\right)$ is contained in $Z_{\infty}\left(G^{\mathfrak{N}}\right)$ and $\langle x\rangle$ is weakly s-supplemented in $G^{\mathfrak{N}}$ for every $x \in$ $\mathcal{P}_{4}\left(F^{*}\left(G^{\mathfrak{N}}\right)\right)$. If $G^{\mathfrak{N}}<G$, then the minimal choice of $G$ implies that $G^{\mathfrak{N}}$ is nilpotent and so $F^{*}\left(G^{\mathfrak{N}}\right)=G^{\mathfrak{N}}$. Thus, by Proposition 3.7, $G$ is nilpotent. This contradiction shows that $G^{\mathfrak{N}}=G$ and so $G / N$ is not nilpotent for any proper normal subgroup of $G$.

(2) $Z_{\infty}(G)=Z(G)$

By [5, Corollary 3.2.9], $Z_{\infty}(G) \cap G^{\mathfrak{N}} \subseteq Z\left(G^{\mathfrak{N}}\right)$. Since $G^{\mathfrak{N}}=G, Z_{\infty}(G)=Z(G)$.

(3) $P \leq Z(G)$.

Obviously, $P$ is normal in $G$. Let $Q$ be a Sylow $q$-subgroup of $G$, where $q \neq p$ is a prime dividing $|G|$. Then $P Q$ is a subgroup of $G$. We claim that $P Q$ is $p$-nilpotent. In fact, by the hypothesis and Lemma 2.3, $\mathcal{P}_{p}(P)=\mathcal{P}_{p}(P Q) \subseteq P Q \cap Z_{\infty}(G) \subseteq Z_{\infty}(P Q)$. By the hypothesis and Lemma 2.4(1), we also see that $\langle x\rangle$ is weakly s-supplemented in $P Q$ for every element $x$ of $\mathcal{P}_{4}(P Q)$. It follows from Lemma 3.5 that $P Q$ is $p$-nilpotent. Therefore, $Q$ is normal in $P Q$ and so $P Q=P \times Q$. Consequently, $O^{p}(G) \leq C_{G}(P)$ and thereby $G / C_{G}(P)$ is a $p$-group. Now by using our claim $(1)$, we obtain that $C_{G}(P)=G$, that is, $P \leq Z(G)$.

(4) Final contradiction.

By our claim (3) and Lemma 2.6(4), $F^{*}(G / P)=F^{*}(G) / P=F / P$. Obviously $2 \nmid$ $|F / P|$. Suppose that $q$ is an arbitrary prime dividing $|F / P|$ and $T / P$ is a subgroup of $F / P$ of order $q$. Then there exists an element $x \in T$ of order $q$ such that $T / P=\langle x\rangle P / P$. By the hypothesis and our claim (2), $x \in Z_{\infty}(G)=Z(G)$. Hence $\langle x\rangle P / P \subseteq Z(G / P)$. This shows that $G / P$ satisfies the hypothesis. The minimal choice of $G$ implies that $G / P$ is nilpotent, which contradicts (1). This final contradiction completes the proof.

Now, by using the above lemmas and proposition, we can prove the following theorem.

THEOREM 3.9. Let $\mathfrak{F}$ be a saturated formation containing $\mathfrak{N}$. Suppose that $G$ contains a normal subgroup $E$ such that $G / E \in \mathfrak{F}$ and $\langle x\rangle$ is weakly s-supplemented in $G$ for every $x \in \mathcal{P}_{4}\left(F^{*}(E)\right)$. Then $G \in \mathfrak{F}$ if and only if every element of $\mathcal{P}\left(F^{*}(E)\right)$ is contained in $Z_{\infty}^{\mathfrak{F}}(G)$ 
Proof. The necessity part is obvious. We only need to prove the sufficiency part. Obviously, $G^{\mathfrak{F}} \subseteq E$. Then by Lemma 2.6, $F^{*}\left(G^{\mathfrak{F}}\right) \subseteq F^{*}(E)$. By [5, Corollary 3.2.9], $Z_{\infty}^{\mathfrak{F}}(G) \cap G^{\mathfrak{F}} \subseteq Z\left(G^{\mathfrak{F}}\right) \subseteq Z_{\infty}\left(G^{\mathfrak{F}}\right)$. Consequently, every element of $\mathcal{P}\left(F^{*}\left(G^{\mathfrak{F}}\right)\right)$ is contained in $Z_{\infty}\left(G^{\mathfrak{F}}\right)$. By the hypothesis and Lemma $2.4,\langle x\rangle$ is weakly s-supplemented in $G^{\mathfrak{F}}$ for every $x \in \mathcal{P}_{4}\left(F^{*}\left(G^{\mathfrak{F}}\right)\right)$. By applying Lemma 3.8, we see that $G^{\mathfrak{F}}$ is nilpotent and so $F^{*}\left(G^{\mathfrak{F}}\right)=G^{\mathfrak{F}}$. Now by Proposition 3.7, we deduce that $G \in \mathfrak{F}$. This completes the proof.

4. Some applications. It is clear that all subgroups, no matter whether they are normal subgroups, c-normal subgroups, c-supplemented subgroups, s-permutable subgroups or weakly s-permutable subgroups, are weakly s-supplemented subgroups.

In the literature [11], a subgroup $H$ of a group $G$ is said to be $p$-nilpotent quotientsupplemented in $G$ if there exists a subgroup $T$ of $G$ such that $H T=G$ and $T / T \cap H_{G}$ is $p$-nilpotent.

It is obvious that if $H$ has a $p$-nilpotent supplement in $G$, then $H$ is a $p$-nilpotent quotient-supplemented in $G$. We now claim that the converse statement also holds. In fact, if $H$ is a $p$-nilpotent quotient-supplemented in $G$, then there exists a subgroup $T$ of $G$ such that $H T=G$ and $T / T \cap H_{G}$ is $p$-nilpotent. If $T$ is $p$-nilpotent, then the assertion is clear. Now, we assume that $T$ is not $p$-nilpotent. Since the class of the $p$-nilpotent groups is a saturated formation, $T \cap H_{G} \nsubseteq \Phi(T)$. Hence, there exists a maximal subgroup $T_{1}$ of $T$ such that $T=\left(T \cap H_{G}\right) T_{1}$. This implies that $H T_{1}=G$ and $T_{1} / T_{1} \cap H_{G}=T_{1} / T_{1} \cap\left(T \cap H_{G}\right) \simeq T / T \cap H_{G}$ is $p$-nilpotent. If $T_{1}$ is $p$-nilpotent, then $H$ has a $p$-nilpotent supplement $T_{1}$ in $G$. If $T_{1}$ is not $p$-nilpotent, then we continue to use the same argument as above. Since $T$ is a finite group, we can eventually find a subgroup $T_{n}$ of $T$ such that $T_{n}$ is $p$-nilpotent and $H T_{n}=G$.

Recall that a group $G$ of order $|G|=p_{1}{ }^{\alpha_{1}} p_{2}{ }^{\alpha_{2}} \ldots p_{n}{ }^{\alpha_{n}}$, where $p_{1}>p_{2}>\cdots>p_{n}$, is said to satisfy the Sylow tower property (see [20, p. 5]) if $G$ has a normal subgroup of order $p_{1}{ }^{\alpha_{1}} p_{2}{ }^{\alpha_{2}} \ldots p_{i}{ }^{\alpha_{i}}$ for every $i \in\{1,2, \ldots, n-1\}$.

Now, by applying Theorem 3.3, we can obtain the following corollaries.

COROLLARY 4.1. Let $\mathfrak{F}$ be the class of all groups satisfying the Sylow tower property. Suppose that a group $G$ has a normal subgroup $E$ such that $G / E \in \mathfrak{F}$. If all subgroups $\langle x\rangle$ of prime order or order 4 (if the Sylow p-subgroup $P$ of $E$ is a non-abelian 2-group) are weakly s-supplemented in $G$, then $G \in \mathfrak{F}$.

Proof. Let $p$ be the smallest prime number dividing $|G|$. Then $(|G|, p-1)=1$. Since $G / E$ satisfies the Sylow tower property, $G / E$ is $p$-nilpotent. Now, it is obvious that $G$ satisfies the hypothesis of Theorem 3.3 for $\mathfrak{H}_{\mathfrak{p}}$. Hence, $G$ must be $p$-nilpotent. Let $T$ be a normal Hall $p^{\prime}$-subgroup of $G$. Then, it can be easily seen that $T$ with respect to $T \cap E$ also satisfies the hypothesis. By induction, we have that $T \in \mathfrak{F}$. This implies that $G \in \mathfrak{F}$.

COROLlARY 4.2. Let $p$ be a prime and $G$ a group with $\left(|G|,(p-1)\left(p^{2}-1\right) \cdots\left(p^{n}-\right.\right.$ $1))=1$, for some integer $n \geq 1$. Suppose that a group $G$ has a normal subgroup $E$ such that $G / E$ is p-nilpotent. If there exists a subgroup $D$ of $E$ such that $1<|D|<p^{n+1}$ and every subgroup $H$ of $E$ with $|H|=|D|$ has a p-nilpotent supplement in $G$, then $G$ is p-nilpotent.

Proof. If $p>2$, then it is clear that $G$ is $p$-nilpotent by Theorem 3.3. We now consider the case $p=2$. Suppose that $K$ is a subgroup of $E$ with $|K|=2|D|$. Then there exists a subgroup $L$ such that $L<K$ and $|L|=|D|$. By the hypothesis, $L$ has a 
$p$-nilpotent supplement $T$ in $G$. Then, it is obvious that $T$ is also a $p$-nilpotent supplement of $K$ in $G$. Thus, by Theorem 3.3, $G$ is $p$-nilpotent.

The following results now follow directly from Theorem 3.3 or the above corollaries.

Corollary 4.3 (Miao, Guo, Shum [11, Theorem 3.1]). Let $G$ be a group and $p$ a prime of $|G|$ such that $\left(|G|, p^{2}-1\right)=1$. Then $G$ is p-nilpotent if and only if there exists a normal subgroup $E$ of $G$ such that $G / E$ is p-nilpotent and each subgroup of $E$ of order $p^{2}$ has a p-nilpotent quotient-supplement in $G$.

Corollary 4.4 (Miao, Guo, Shum [11, Theorem 3.3]). Let $G$ be a group and $(|G|, 21)=1$. Then $G$ is 2-nilpotent if and only if each subgroup of $G$ of order 8 has a 2-nilpotent quotient-supplement in $G$.

Corollary 4.5 (Ramadan, Ezzat Mohaemed, Heliel [12, Lemma 3.8]). Let $p$ be the smallest prime divisor dividing the order of a group $G$. If $\langle x\rangle$ is c-normal in $G$ for every $x \in \mathcal{P}_{p}^{*}(G)$, then $G$ is p-nilpotent.

COROLlary 4.6 (Zhong, Li [22, Theorem 2.3]). Let $G$ be a group and $p$ the smallest prime divisor dividing $|G|$. Suppose that there exists a normal subgroup $E$ of $G$ such that $G / E$ is p-nilpotent. If $\langle x\rangle$ is c-supplemented in $G$ for every $x \in \mathcal{P}_{p}^{*}(E)$, then $G$ is p-nilpotent.

Corollary 4.7 (Xie, Shi, Hu [21, Theorem 3.4]). Let $p$ be a prime number and $G$ a group with $(|G|, p-1)=1$. If $\langle x\rangle$ is weakly s-supplemented in $G$ for every $x \in \mathcal{P}_{p}^{*}(G)$, then $G$ is p-nilpotent.

The following known result follows directly from Lemma 3.5.

COROllary 4.8 (Lam, Shum, Guo [8]). If $p$ is an odd prime and every element of $\mathcal{P}_{p}(G)$ is contained in $Z_{\infty}(G)$, then $G$ is p-nilpotent.

The following known result now follows directly from Theorem 3.9.

COROLlaRY 4.9 (Wang [17, Theorem 3.1]). Suppose that a group G has a normal subgroup $E$ such that $G / E$ is nilpotent and $\langle x\rangle$ is c-normal in $G$ for every $x \in \mathcal{P}_{4}(E)$. Then $G$ is nilpotent if and only if every element of $\mathcal{P}\left(F^{*}(E)\right)$ is contained in $Z_{\infty}^{\mathfrak{F}}(G)$.

COROLlARY 4.10 (Ballester-Bolinches, Wang [1, Theorem 3.1]). Let $\mathfrak{F}$ be a saturated formation containing $\mathfrak{N}$. Suppose that $G$ is a group and $\langle x\rangle$ is c-normal in $G$ for every $x \in \mathcal{P}_{4}\left(G^{\mathfrak{F}}\right)$. Then $G \in \mathfrak{F}$ if and only if every element of $\mathcal{P}\left(G^{\mathfrak{F}}\right)$ is contained in $Z_{\infty}^{\mathfrak{F}}(G)$.

COROLlARY 4.11 (Li [9, Theorem 1]). Let $\mathfrak{F}$ be a saturated formation containing $\mathfrak{N}$ and $G$ a group. Then $G \in \mathfrak{F}$ if and only if there exists a normal solvable subgroup $E$ such that $G / E \in \mathfrak{F}$ and every element of $\mathcal{P}(F(E))$ is contained in $Z_{\infty}^{\mathfrak{F}}(G)$ and $\langle x\rangle$ is c-normal in $G$ for every $x \in \mathcal{P}_{4}(F(E))$.

Corollary 4.12 (Ballester-Bolinches, Wang [1, Corollary 3.2]). Let $G$ be a group such that $\langle x\rangle$ is c-normal in $G$ for every $x \in \mathcal{P}_{4}\left(F^{*}(G)\right)$. If every element of $\mathcal{P}\left(F^{*}(G)\right)$ is contained in $Z_{\infty}(G)$, then $G$ is nilpotent. 
Corollary 4.13 (Li, Wang [10, Theorem 4.5]). Suppose that E is a normal subgroup of a group $G$ such that $G / E$ is nilpotent. Suppose that $\langle x\rangle$ is $\pi$-quasi-normal in $G$ for every $x \in \mathcal{P}_{4}\left(F^{*}(E)\right)$. Then $G$ is nilpotent if and only if every element of $\mathcal{P}\left(F^{*}(E)\right)$ is contained in $Z_{\infty}(G)$.

Corollary 4.14 (Li, Wang [10, Theorem 4.6]). Let $\mathfrak{F}$ be a saturated formation containing $\mathfrak{N}$. Suppose that $\langle x\rangle$ is $\pi$-quasi-normal in a group $G$ for every $x \in \mathcal{P}_{4}\left(G^{\mathfrak{F}}\right)$. Then $G \in \mathfrak{F}$ if and only if every element of $\mathcal{P}\left(G^{\mathfrak{F}}\right)$ is contained in $Z_{\infty}^{\mathfrak{F}}(G)$.

Corollary 4.15 (Zhong, Li [22, Theorem 2.5]). Suppose that $p$ is a prime and $G$ is a group. If every element of $\mathcal{P}_{p}\left(G^{\mathfrak{N}}\right)$ is contained in $Z_{\infty}(G)$ and $\langle x\rangle$ is c-supplemented in $G$ for every $x \in \mathcal{P}_{4}\left(G^{\mathfrak{N}}\right)$, then $G$ is p-nilpotent.

COROLlary 4.16 (Zhong, Li [22, Theorem 2.6]). Suppose that a group $G$ has a normal subgroup $E$ such that $G / E$ is nilpotent. If every element of $\mathcal{P}\left(F^{*}(E)\right)$ is contained in $Z_{\infty}(G)$ and $\langle x\rangle$ is c-supplemented in $G$ for every $x \in \mathcal{P}_{4}\left(F^{*}(E)\right)$, then $G$ is nilpotent.

Corollary 4.17 (Wang, Li, Wang [19, Theorem 4.4]). Let $\mathfrak{F}$ be a saturated formation containing $\mathfrak{N}$ and $G$ a group. Suppose that $\langle x\rangle$ is c-supplemented in $G$ for every $x \in \mathcal{P}_{4}\left(G^{\mathfrak{F}}\right)$. Then $G \in \mathfrak{F}$ if and only if every element of $\mathcal{P}\left(G^{\mathfrak{F}}\right)$ is contained in $Z_{\infty}^{\mathfrak{F}}(G)$

Corollary 4.18 (Wang, Li, Wang [19, Theorem 3.3]). Suppose that a group G has a normal subgroup $E$ such that $G / E$ is nilpotent. Suppose that $\langle x\rangle$ is c-supplemented in $G$ for every $x \in \mathcal{P}_{4}\left(F^{*}(E)\right)$. Then $G$ is nilpotent if and only if every element of $\mathcal{P}\left(F^{*}(E)\right)$ is contained in $Z_{\infty}^{\mathfrak{F}}(G)$.

Corollary 4.19 (Wang, Li, Wang [19, Theorem 4.5]). Let $\mathfrak{F}$ be a saturated formation containing $\mathfrak{N}$. Suppose that $\langle x\rangle$ is c-supplemented in $G$ for every $x \in$ $\mathcal{P}_{4}\left(F^{*}\left(G^{\mathfrak{F}}\right)\right)$. Then $G \in \mathfrak{F}$ if and only if every element of $\mathcal{P}\left(F^{*}\left(G^{\mathfrak{F}}\right)\right)$ is contained in $Z_{\infty}^{\mathfrak{F}}(G)$.

ACKNOWLEDGEMENTS. The authors cordially thank the referee for his/her helpful comments. The research was supported by an NNSF grant of China (Grant \#11071229).

\section{REFERENCES}

1. A. Ballester-Bolinches and Y. Wang, Finite groups with some c-normal minimal subgroups, J. Pure Appl. Algebra 153 (2000), 121-127. $15-17$.

2. J. Buckley, Finite groups whose minimal subgroups are normal, Math. Z. 116 (1970),

3. K. Doerk and T. Hawkes, Finite solvable groups (Walter de Gruyter, New York, 1992).

4. W. Guo, The influence of minimal subgroups on the structure of finite groups, Southeast Asian Bull. Math. 22 (1998), 287-290.

5. W. Guo, The Theory of classes of groups (Science Press-Kluwer Academic Publishers, Beijing-New York-Dordrecht-Boston-London, 2000).

6. B. Huppert, Endliche Gruppen I (Springer, Berlin-Heidelberg-New York, 1979).

7. B. Huppert and N. Blackburn, Finite groups III (Springer, Berlin-New York, 1982).

8. C. M. Lam, K. P. Shum and W. Guo, A generalized Ito theorem on $p$-nilpotent groups, Pure Math. Appl. 11 (2000), 593-596. 
9. Y. Li, Some notes on the minimal subgroups of Fitting subgroups of finite groups, $J$. Pure Appl. Algebra 171 (2002), 289-294.

10. Y. Li and Y. Wang, On $\pi$-quasinormally embedded subgroups of finite group, J. Algebra 281 (2004), 109-123.

11. L. Miao, W. Guo and K. P. Shum, New criteria for $p$-nilpotency of finite groups, Commun. Algebra 35 (2007), 965-974.

12. M. Ramadan, M. E. Mohamed and A. A. Heliel, On c-normality of certain subgroups of prime power order of finite groups, Arch. Math. 85 (2005), 203-210.

13. D. J. S. Robinson, A Course in theory of group (Spinger, New York, 1982).

14. L. A. Shemetkov, Formations of finite groups (Nauka, Moscow, 1978).

15. A. N. Skiba, On weakly s-permutable subgroups of finite groups, J. Algebra 315 (2007), 192-209.

16. Y. Wang, c-Normality of groups and its properties, J. Algebra 180 (1996), 954-965.

17. Y. Wang, The influence of minimal subgroups on the structure of finite group, Acta. Math. Sinica (English series) 16 (2000), 63-70.

18. Y. Wang, Finite groups with some subgroup of Sylow subgroups c-supplemented, $J$. Algebra 224 (2000), 467-478.

19. Y. Wang, Y. Li and J. Wang, Finite groups with c-supplemented minimal subgroups, Algebra Colloquium 10(3) (2003), 413-425.

20. M. Weinstein (Editor), in Between Nilpotent and Solvable (Bray H. G., Deskins W. E., Johnson D., Humphreys J. F., Puttaswamaiah B. M., Venzke P. and Walls G. L., Editors) (Polygonal Publishing House, NJ, USA, 1982).

21. F. Xie, L. Shi and B. Hu, Some new criteria for $p$-nilpotency of finite groups, Math. Sci. Res. J. 12(2) (2008), 38-43.

22. X. Zhong and S. Li, On c-supplemented minimal subgroups of finite groups, Southeast Asian Bull. Math. 28 (2004), 1141-1148. 